\title{
A BOUND ON THE ERROR IN PLATE THEORY*
}

\author{
BY \\ R. P. NORDGREN \\ Shell Development Company, Houston
}

Introduction. A solution to a boundary value problem in the classical two-dimensional theory of plates is generally accepted as an approximate solution to a corresponding boundary value problem in the three-dimensional theory of elasticity provided that the plate is sufficiently thin. This conclusion is supported by several exact solutions for plates in the theory of elasticity [1] and by the fact that the equations of plate theory can be obtained from the equations of elasticity theory as the leading terms in parametric expansions [2], [3]. Further, Morgenstern [4] has shown that the stresses and strains obtained from a solution in plate theory converge in a mean-square sense to a solution in elasticity theory as the plate thickness approaches zero. Related theorems on meansquare convergence of parametric expansions for a problem in beam theory are stated by Babuška and Prager [5].

In the present paper we derive an explicit expression for the mean-square error in the components of stress obtained from a solution in plate theory with respect to the exact solution of a corresponding problem in the theory of elasticity. In addition, a precise bound is given for the relative mean-square error. The derivation employs the hypersphere theorems of Prager and Synge [6] in the theory of elasticity. In the course of the derivation the equations of plate theory are obtained in two ways by minimization of portions of both the potential energy and the complementary energy. The general expression obtained for the error contains only quantities which are available from a solution in plate theory.

Our results and the previous investigations of convergence [4], [5] show that the relative mean-square error in plate theory is proportional to the thickness of the plate in general. This is somewhat surprising since the exact solutions for plates in elasticity theory [1] give a relative error proportional to the square of the thickness. This form for relative error also is indicated by the parametric expansions [2]. The discrepancy in our result can be attributed to the expression obtained for the components of transverse shear stress, which differs from the classical expression. We have been unable to derive the classical expression by the present method. (See note added in proof at the end of this paper.)

1. Function space concepts in elasticity. We consider a three-dimensional elastic body $R$ bounded by a closed surface $S$. With reference to a system of rectangular Cartesian coordinates $x_{i}(i=1,2,3)$, the field equations of the linear theory of elasticity read as follows [1]:

equilibrium (in the absence of body force)

\footnotetext{
* Received January 5, 1970.
} 


$$
\sigma_{i, i}=0, \quad \sigma_{i i}=\sigma_{i i}
$$

strain-displacement

$$
e_{i j}=\frac{1}{2}\left(u_{i, i}+u_{i, i}\right)
$$

generalized Hooke's law

$$
e_{i i}=A_{i j k l} \sigma_{k l}, \quad \sigma_{i j}=B_{i j k l} e_{k l},
$$

where $\sigma_{i j}$ is the stress tensor, $e_{i j}$ is the strain tensor and $u_{i}$ is the displacement vector. The elastic constants $A_{i j k l}$ and $B_{i j k l}$ satisfy

$$
\begin{aligned}
& A_{i j k l}=A_{i i k l}=A_{i j l k}=A_{k l i j} \\
& B_{i j k l}=B_{i i k l}=B_{i j l k}=B_{k l i j} \\
& A_{i j k l} B_{k l m n}=\frac{1}{2}\left(\delta_{i m} \delta_{i n}+\delta_{i n} \delta_{i m}\right)
\end{aligned}
$$

and are such that the strain energy density $W$ is positive definite where

$$
W=\frac{1}{2} A_{i j k l} \sigma_{i j} \sigma_{k l}=\frac{1}{2} B_{i j k l} e_{i j} e_{k l} \text {. }
$$

We consider boundary value problems where the boundary $S$ is divided into two parts $S_{u}$ and $S_{\bullet}$ on which boundary conditions are

$$
\begin{aligned}
& u_{i}=u_{i}^{*} \text { on } S_{u}, \\
& \sigma_{i}=\sigma_{i} n_{i}=\sigma_{i}^{*} \text { on } S_{\sigma},
\end{aligned}
$$

where $u_{i}^{*}$ is the prescribed displacement vector on $S_{u}, \sigma_{i}^{*}$ is the prescribed stress vector on $S_{\sigma}$ and $n_{i}$ is the outward unit normal vector to $S$. Somewhat more general linear boundary conditions may be treated without difficulty.

Following Prager and Synge [6] and Synge [7], for states of stress $\sigma_{i}$ such that

$$
\int_{R} A_{i j k l} \sigma_{i j} \sigma_{k l} d V<\infty
$$

we consider the vector space with componentwise addition and inner product defined by

$$
\boldsymbol{\sigma}^{\prime} \cdot \boldsymbol{\sigma}^{\prime \prime}=\int_{R} A_{i j k l} \sigma_{i ;}^{\prime} \sigma_{k l}^{\prime \prime} d V,
$$

where $\boldsymbol{\sigma}^{\prime}$ and $\boldsymbol{\sigma}^{\prime \prime}$ denote two states of stress with components $\sigma_{i j}^{\prime}$ and $\sigma_{i i}^{\prime \prime}$ which satisfy (1.8). The norm of $\delta$ is defined as

$$
\|\boldsymbol{\sigma}\|=(\boldsymbol{\sigma} \cdot \boldsymbol{d})^{1 / 2}
$$

It can be verified that the foregoing definitions satisfy the basic postulates for a linear vector space [8].

In what follows unprimed quantities denote the actual solution to the boundary value problem (1.1) to (1.7). For this same boundary value problem, primed quantities satisfy (1.1), (1.3) and (1.7), and double-primed quantities satisfy (1.2), (1.3) and (1.6).

1 Commas denote partial differentiation and repeated indices imply summation.

2 The Kronecker delta $\delta_{i j}$ takes the value 1 if $i=j$ and 0 if $i \neq j$. 
Then, with use of the divergence theorem, it follows that

$$
\boldsymbol{\sigma}^{\prime} \cdot \boldsymbol{\sigma}^{\prime \prime}=\int_{S_{凶}} u_{i}^{*} \sigma_{i}^{\prime} d S+\int_{S_{\bullet}} u_{i}^{\prime \prime} \sigma_{i}^{*} d S .
$$

Since $\boldsymbol{\sigma}^{\prime}$ or $\boldsymbol{\delta}^{\prime \prime}$ in (1.11) can be replaced by $\boldsymbol{\delta}$, we have

$$
\boldsymbol{d} \cdot \boldsymbol{d}-\boldsymbol{d}^{\prime} \cdot \boldsymbol{d}-\boldsymbol{d} \cdot \boldsymbol{d}^{\prime \prime}+\boldsymbol{\delta}^{\prime} \cdot \boldsymbol{d}^{\prime \prime}=0,
$$

which is equivalent to

$$
\left\|\boldsymbol{\delta}-\boldsymbol{\delta}_{\boldsymbol{A}}\right\|=E
$$

where

$$
\boldsymbol{\sigma}_{A}=\frac{1}{2}\left(\boldsymbol{d}^{\prime}+\boldsymbol{\sigma}^{\prime \prime}\right), \quad E=\left\|\frac{1}{2}\left(\boldsymbol{\sigma}^{\prime}-\boldsymbol{\sigma}^{\prime \prime}\right)\right\| .
$$

Thus, if $\boldsymbol{\delta}_{A}$ is considered an approximation to $\boldsymbol{\delta}$, (1.12) gives the error $E$ of this approximation in the integral-square norm (1.10). In vector space geometry (1.12) implies that $\delta$ lies on a hypersphere with center at $\delta_{A}$ and radius $E$.

In order to investigate relative error we recall the inequalities [7]

$$
\left\|\boldsymbol{\sigma}_{A}\right\|-E \leq\|\boldsymbol{\sigma}\| \leq\left\|\sigma_{A}\right\|+E,
$$

which follow from (1.12) and the triangle inequality. By (1.13), assuming that $E<\left\|\boldsymbol{\delta}_{A}\right\|$, we have the following bounds on the relative error $E /\|\boldsymbol{\sigma}\|$ :

$$
\frac{E}{\left\|\boldsymbol{\sigma}_{A}\right\|+E} \leq \frac{E}{\|\boldsymbol{\sigma}\|} \leq \frac{E}{\left\|\sigma_{A}\right\|-E} .
$$

Since, by (1.12),

$$
\left\|\boldsymbol{\sigma}_{A}\right\|^{2}=\boldsymbol{\sigma}^{\prime} \cdot \boldsymbol{\sigma}^{\prime \prime}+E^{2}
$$

(1.14) can be written as

$$
\eta\left(\sqrt{ }\left(1+\eta^{2}\right)-\eta\right) \leq E /\|\boldsymbol{\alpha}\| \leq \eta\left(\sqrt{ }\left(1+\eta^{2}\right)+\eta\right)
$$

where

$$
\eta=E /\left(\boldsymbol{d}^{\prime} \cdot \boldsymbol{\sigma}^{\prime \prime}\right)^{1 / 2}
$$

From (1.16) we see that $\eta$ is the leading term in an expansion of the relative error in powers of $\eta$.

A convenient method of obtaining $\boldsymbol{\delta}^{\prime}$ and $\boldsymbol{\delta}^{\prime \prime}$ follows from the easily verified relation [7]

$$
E^{2}=\frac{1}{2}\left(V_{c}+V_{p}\right)
$$

where

$$
\begin{aligned}
& V_{c}=\int_{R} \frac{1}{2} A_{i j k l} \sigma_{i j}^{\prime} \sigma_{k l}^{\prime} d V-\int_{S_{s}} u_{i}^{*} \sigma_{i}^{\prime} d S \\
& V_{p}=\int_{R} \frac{1}{2} A_{i j k l} \sigma_{i j}^{\prime \prime} \sigma_{k l}^{\prime \prime} d V-\int_{S_{\sigma}} u_{i}^{\prime \prime} \sigma_{i}^{*} d S
\end{aligned}
$$

are the complementary energy and the potential energy, respectively. Thus, the usual methods of energy minimization can be interpreted as minimizations of the error $E$. 
2. Boundary value problems for plates. We consider an elastic body in the form of a plate bounded by the faces $x_{3}= \pm h$ and the edge surface $\widetilde{S}$ which is generated by normals to the middle plane $S$ through the edge curve $C$. Attention will be confined to plates of constant thickness $2 h$ although there is no difficulty in extending the results to variable thickness.

Stress boundary conditions are imposed on the faces of the plate. For simplicity we consider the faces to be subject only to prescribed normal components of stress, i.e.

$$
\begin{gathered}
\sigma_{13}=\sigma_{23}=0 \quad \text { on } x_{3}= \pm h, \\
\sigma_{33}\left(x_{1}, x_{2}, h\right)=\sigma_{3}^{+}\left(x_{1}, x_{2}\right), \quad \sigma_{33}\left(x_{1}, x_{2},-h\right)=-\sigma_{3}^{-}\left(x_{1}, x_{2}\right) .
\end{gathered}
$$

The edge surface of the plate is divided into two parts $\widetilde{S}_{u}$ and $\widetilde{S}_{\sigma}$ which have displacement and stress boundary conditions of the form (1.6) and (1.7), namely

$$
\begin{gathered}
u_{i}=u_{i}^{*} \text { on } \widetilde{S}_{u}, \\
\sigma_{i \alpha} n_{\alpha}=\sigma_{i}^{*} \text { on } \widetilde{S}_{\sigma},
\end{gathered}
$$

where $n_{\alpha}$ is the normal to $C$ in the middle plane and Greek indices take values 1 and 2 . Here, we restrict $u_{i}^{*}$ and $\sigma_{i}^{*}$ to the forms

$$
\begin{aligned}
u_{\alpha}^{*} & =\beta_{\alpha}^{*} x_{3}, \\
u_{3}^{*} & =w^{*}+g^{*} x_{3}^{2}, \\
\sigma_{\alpha}^{*} & =\frac{3}{2 h^{3}} M_{\alpha}^{*} x_{3}, \\
\sigma_{3}^{*} & =\frac{3}{4 h}\left(1-\frac{x_{3}^{2}}{h^{2}}\right) Q^{*},
\end{aligned}
$$

where $\beta_{\alpha}^{*}, w^{*}, g^{*}, M_{\alpha}^{*}$ and $Q^{*}$ are independent of $x_{3}$. The constants in (2.6) and (2.7) have been chosen so that $M_{\alpha}^{*}$ and $Q^{*}$ represent the stress couple and the shear stress resultant, respectively, i.e.,

$$
\int_{-h}^{h} \sigma_{\alpha}^{*} x_{3} d x_{3}=M_{\alpha}^{*}, \quad \int_{-h}^{h} \sigma_{3}^{*} d x_{3}=Q^{*} .
$$

We now have a boundary value problem in the theory of elasticity for a plate with boundary conditions (2.1) to (2.7). The aim of the theory of plates is to reduce this three-dimensional boundary value problem to a two-dimensional problem involving quantities which are independent of $x_{3}$. Here we will derive the equations of the classical theory of plates by consideration of both the complementary energy and the potential energy (1.18). Then, (1.12) will yield an expression for the error in an approximation for the stresses in the plate. Since the well-known edge conditions for the classical theory of plates [1] are not as general as (2.4) to (2.7), we expect to obtain restrictions on the quantities on the right-hand side of these equations.

For convenience we restrict attention to anisotropic plates for which the middle plane is a plane of elastic symmetry. Then (1.3) takes the form [1]

$e_{\alpha \beta}=A_{\alpha \beta \gamma \delta} \sigma_{\gamma \delta}+A_{\alpha \beta 33} \sigma_{33}, \quad e_{33}=A_{33 \gamma \delta} \sigma_{\gamma \delta}+A_{3333} \sigma_{33}, \quad \epsilon_{\alpha^{2}}=2 A_{\alpha 3 \beta 3} \sigma_{\beta 3}$,

and

$\sigma_{\alpha \beta}=B_{\alpha \beta \gamma \delta} e_{\gamma \delta}+B_{\alpha \beta 33} e_{33}, \quad \sigma_{33}=B_{33 \gamma \delta} e_{\gamma \delta}+B_{3333} e_{33}, \quad \sigma_{\alpha 3}=2 B_{\alpha 3 \beta 3} e_{\beta 3}$. 
3. Potential energy. Here, (1.2), (1.3) and (1.6) must be satisfied and $V_{p}$ in (1.18) is to be minimized. Guided by the three-dimensional solution for pure bending of a plate [1], we assume displacements of the form

$$
u_{\alpha}^{\prime \prime}=-w_{. \alpha} x_{3}, \quad u_{3}^{\prime \prime}=w+g x_{3}^{2},
$$

where $w$ and $g$ are independent of $x_{3}$. The first of (3.1) represents the classical Kirchhoff assumption that normals to the undeformed middle plane remain normal to the deformed middle surface.

By (1.2) and (3.1), the strains are

$$
f_{\alpha \beta}^{\prime \prime}=-u_{, \alpha \beta} \cdot x_{3} . \quad \rho_{\alpha 3}^{\prime \prime}=\frac{1}{2} g_{, \alpha} x_{3}^{2}, \quad e_{33}^{\prime \prime}=2 g \cdot x_{3} .
$$

If we take

$$
g=\frac{B_{33 \alpha \beta}}{2 B_{3333}} w{ }_{\alpha \beta}
$$

then, by $(2.10)$ and $(3.2)$

$$
\sigma_{33}^{\prime \prime}=0
$$

as in the solution for pure bending. The discrepancy between (2.1) and (3.4) is admissible since $\sigma_{i j}^{\prime \prime}$ need not satisfy stress boundary conditions on $S_{\sigma}$. By (2.10), (3.2) and (3.3), the remaining stresses are given by

$$
\sigma_{\alpha \beta}^{\prime \prime}=-\widetilde{B}_{n \beta, \delta} w_{, \gamma \delta} x_{3}, \quad \sigma_{\alpha 3}^{\prime \prime}=B_{\alpha 3 \beta 3} g_{. \beta} r_{3}^{2},
$$

where

$$
\widetilde{B}_{\sigma \beta \gamma \delta}=B_{\alpha \beta \gamma \dot{0}}-B_{\alpha \beta 33} B_{\gamma \delta 33} / B_{3333} .
$$

The displacement. (3.1) must satisfy (2.2) with (2.4) and (2.5), i.e.,

$$
\left.\begin{array}{rl}
w & =w^{*}(s), \\
\frac{\partial w}{\partial n} & =-\beta_{\alpha}^{*}(s) n_{\alpha}, \\
g & =g^{*}(s), \\
\beta_{\alpha}^{*} & =\frac{\partial w^{*}}{\partial s}
\end{array}\right\} \text { on } C .
$$

where $s$ is the arc length on $C$ and $\partial w / \partial n$ denotes the normal derivative of $w$. Since $g$ is determined by (3.3), the function $g^{*}(s)$ cannot be specified arbitrarily but must be compatible with (3.3). Thus, $g^{*}(s)$ is not known until $w$ has been obtained for a particular problem. ${ }^{4}$ The last of (3.6) is a compatability requirement on $w^{*}$ and $\beta_{\alpha}^{*}$ as a consequence of the Kirchhoff hypothesis.

By (2.1), (2.3), (2.6), (2.7), (3.2) and (3.3), the potential energy (1.18) can be written

3 The components of the $\epsilon$-symbol $\epsilon_{\alpha \beta}$ have the values $\epsilon_{11}=\epsilon_{2 i}=0, \epsilon_{12}=1$ and $\epsilon_{21}=-1$.

- It is possible to avoid this unpleasantness by not invoking (3.3) and leaving $g$ as a basic variable akin to $w$. However, classical plate theory does not result. 
as

$$
V_{p}=V_{p}^{(0)}+V_{p}^{(2)} h^{2}
$$

where

$$
\begin{gathered}
V_{\nu}^{(0)}=\int_{s}\left[\frac{1}{3} h^{3} \tilde{B}_{\alpha \beta \gamma \delta} w_{, \alpha \beta} w_{, \gamma \delta}-p w\right] d x_{1} d x_{2}+\int_{c_{\sigma}}\left[M_{\alpha}^{*} w_{, \alpha}-Q^{*} w\right] d s, \\
V_{\bullet}^{(2)}=\int_{s}\left[\frac{1}{5} h^{3} B_{\alpha 3 \beta 3} g_{, \alpha} g_{, \beta}-p g\right] d x_{1} d x_{2}-\frac{1}{5} \int_{c_{\odot}} g Q^{*} d s, \\
p=\sigma_{3}^{+}+\sigma_{3}^{-} .
\end{gathered}
$$

On minimization of $V_{p}^{(0)}$ by standard techniques of the calculus of variations we obtain the Euler equation

$$
\frac{2}{3} h^{3} \widetilde{B}_{\alpha \beta \gamma \delta} w_{, \alpha \beta \gamma \delta}=p \text { in } S
$$

and the natural boundary conditions

$$
\left.\begin{array}{c}
M_{N}=M_{N}^{*} \\
Q_{\alpha} n_{\alpha}-\frac{\partial M_{T}}{\partial s}=Q^{*}-\frac{\partial M_{T}^{*}}{\partial s}
\end{array}\right\} \text { on } C .
$$

where

$$
\begin{array}{cl}
M_{N}^{*}=M_{\alpha}^{*} n_{\alpha}, & M_{T}^{*}=\epsilon_{\alpha \beta} M_{\alpha}^{*} n_{\beta}, \\
M_{N}=M_{\alpha \beta} n_{\alpha} n_{\beta}, & M_{T}=\epsilon_{\beta \gamma} M_{\alpha \beta} n_{\alpha} n_{\gamma} . \\
M_{\alpha \beta}=-\frac{2}{3} h^{3} \widetilde{B}_{\alpha \beta \gamma \delta} w_{, \gamma \delta}, & Q_{\alpha}=-\frac{2}{3} h^{3} \widetilde{B}_{\alpha \beta \gamma \delta} w_{, \beta \gamma \delta} .
\end{array}
$$

The quantities $M_{\alpha \beta}$ and $Q_{\alpha}$ may be interpreted as stress couple and transverse shear force resultant, respectively. The subscripts $N$ and $T$ denote normal and tangential components of stress couple on $C$. The derivatives in (3.9) should be understood in the symbolic sense of the theory of generalized functions, i.e., a jump discontinuity in $M_{T}$ gives a Dirac delta symbol for $\partial M_{T} / \partial s$ which represents a concentrated force.

4. Complementary energy. In this approach $V_{c}$ is to be minimized for admissible stresses which meet (1.1) and (1.7). If we assume that the $x_{3}$ variation of stresses is of the form

$$
\begin{aligned}
& \sigma_{\alpha \beta}^{\prime}=\frac{3 x_{3}}{2 h^{3}} M_{\alpha \beta}^{\prime}, \\
& \sigma_{\alpha 3}^{\prime}=\frac{3}{4 h}\left(1-\frac{x_{3}^{2}}{h^{2}}\right) Q_{\alpha}^{\prime}, \\
& \sigma_{33}^{\prime}=\frac{3}{4 h}\left(1-\frac{x_{3}^{2}}{3 h^{2}}\right) x_{3} p+q,
\end{aligned}
$$

where

$$
P=\sigma_{3}^{+}+\sigma_{3}^{-}, \quad q=\frac{1}{2}\left(\sigma_{3}^{+}-\sigma_{3}^{-}\right),
$$

then (1.1) are satisfied provided that

$$
M_{\alpha \beta, \beta}^{\prime}-Q_{\alpha}^{\prime}=0, \quad-Q_{\alpha, \alpha}^{\prime}=p .
$$


Further, (4.1) satisfies (2.1) identically and guided by (3.9) we impose the boundary conditions

$$
M_{N}^{\prime}=M_{N}^{*}, \quad Q_{\alpha}^{\prime} n_{\alpha}-\partial M_{T}^{\prime} / \partial s=Q^{*}-\partial M_{T}^{*} / \partial s \text { on } C .
$$

where

$$
M_{N}^{\prime}=M_{\alpha \beta}^{\prime} n_{\alpha} n_{\beta}, \quad M_{T}^{\prime}=\epsilon_{\beta \gamma} M_{\alpha \beta}^{\prime} n_{\alpha} n_{\gamma}
$$

As mentioned in Sec. 2, classical plate theory requires a restriction on $M_{\alpha}^{*}$ and $Q^{*}$ in (2.6) and (2.7). Namely, we can specify only the two quantities $M_{N}^{*}$ and $Q^{*}-\partial M_{T}^{*} / \partial s$ on $C_{\sigma}$ and we must accept the results of the plate theory solution for $M_{\alpha}^{*}$ and $Q^{*}$. With this restriction on the elasticity problem, the stress (4.1) under (4.2) and (4.3) are admissible as $\sigma_{i j}^{\prime}$ in the formulas of Sec. 1 .

On substitution of (4.1) into (1.18), $V_{c}$ can be written as

$$
V_{c}=V_{c}^{(0)}+V_{c}^{(2)} h^{2}
$$

where

$$
\begin{gathered}
V_{c}^{(0)}=\int_{s} \frac{3}{4 h^{3}} A_{\alpha \beta \gamma \delta} M_{\alpha \beta}^{\prime} M_{\gamma \delta}^{\prime} d x_{1} d x_{2}-\int_{c_{\alpha}}\left[\beta_{\alpha}^{*} M_{\alpha \beta}^{\prime} n_{\beta}+w^{*} Q_{\alpha}^{\prime} n_{\alpha}\right] d s, \\
V_{c}^{(2)}=\int_{s}\left[\frac{3}{5 h^{3}} A_{\alpha \beta 33} M_{\alpha \beta}^{\prime} p+A_{3333}\left(\frac{17 p^{2}}{140 h}+\frac{q^{2}}{h}\right)\right. \\
\left.+\frac{6}{5 h^{3}} A_{\alpha 3 \beta 3} Q_{\alpha}^{\prime} Q_{\beta}^{\prime}\right] d x_{1} d x_{2}-\frac{1}{5} \int_{e_{\sigma}} g^{*} Q_{\alpha}^{\prime} n_{\alpha} d s .
\end{gathered}
$$

In order to minimize $V_{c}^{(0)}$ subject to (4.2), we introduce the Lagrange multiplier $w^{\prime}$ and minimize

$$
V_{c}^{(0)}-\int_{S} w^{\prime}\left(M_{\alpha \beta, \alpha \beta}^{\prime}+p\right) d x_{1} d x_{2} .
$$

By the calculus of variations we obtain the Euler equation

$$
\frac{3}{2 h^{3}} A_{\alpha \beta \gamma \delta} M_{\gamma^{\delta}}^{\prime}+w_{, \alpha \beta}^{\prime}=0 \text { in } S,
$$

which, by (1.4), is equivalent to

$$
M_{\alpha \beta}^{\prime}=-\frac{2 h^{3}}{3} \widetilde{B}_{\alpha \beta \gamma \delta} w_{, \gamma \delta}^{\prime},
$$

where $\widetilde{B}_{\alpha \beta \gamma \delta}$ is defined at (3.5). We also obtain the natural boundary conditions

$$
w^{\prime}=w^{*}(s), \quad \frac{\partial w^{\prime}}{\partial n}=-\beta_{\alpha}^{*}(s) n_{\alpha} \quad \text { on } C_{u},
$$

which agrees with (3.6). Substitution of (4.7) into (4.2) results in

$$
Q_{\alpha}^{\prime}=-\frac{2 h^{3}}{3} \tilde{B}_{\alpha \beta \gamma \delta} w_{, \beta \gamma \delta}^{\prime}
$$


and

$$
\frac{2 h^{3}}{3} \widetilde{B}_{\alpha \beta \gamma \delta} w_{. \alpha \beta \gamma \delta}^{\prime}=p
$$

which is identical with (3.8).

5. Approximate solution and error. Since the field equations and boundary conditions of Secs. 3 and 4 are identical we make no distinction between primed and unprimed quantities $w, M_{\alpha \beta}$ and $Q_{\alpha}$ for the plate. Further, the equations and boundary conditions for $w, M_{\alpha \beta}, Q_{\alpha}$ are easily recognized as those of classical plate theory [1].

In a particular boundary value problem of plate theory, after a solution has been obtained for $w$, then $g^{*}, M_{\alpha}^{*}$ and $Q^{*}$ can be determined from (3.3), (4.3), and (4.7) and (4.9). These values of $g^{*}, M_{\alpha}^{*}$ and $Q^{*}$ together with the prescribed values $\sigma_{3}^{ \pm}\left(x_{1}, x_{2}\right)$, $w^{*}$ and $\beta_{\alpha}^{*} n_{\alpha}$ define a three-dimensional boundary value problem for an elastic plate as discussed in Sec. 2. For this problem we determine the error in the approximate solution of plate theory by the method of Sec. 1 .

For use in the error formula (1.12), by (3.4), (3.5), (4.1), (4.7) and (4.9), the approximate plate stresses are

$$
\begin{aligned}
& \sigma_{\alpha \beta}^{A}=-\widetilde{B}_{\alpha \beta \gamma \delta} w_{. \gamma \delta} x_{3}, \\
& \sigma_{\alpha 3}^{A}=\frac{1}{4}\left[\frac{B_{\alpha 3 \beta 3} B_{33 \gamma \delta}}{B_{3333}} x_{3}^{2}-\widetilde{B}_{\alpha \beta \gamma \delta}\left(h^{2}-x_{3}^{2}\right)\right] w_{, \beta \gamma \delta}, \\
& \sigma_{33}^{A}=\frac{3}{8 h}\left(1-x_{3}^{2} / 3 h^{2}\right) x_{3} p+\frac{1}{2} q,
\end{aligned}
$$

where $p$ and $q$ are defined by (4.1). By (1.12), (3.4), (3.5), (4.1), (4.7) and (4.9) we find that

$$
\begin{array}{r}
E^{2}=\frac{1}{2} \int_{S}\left[\frac{1}{5} h^{5} B_{\alpha 3 \beta 3} g_{. \alpha} g_{. \beta}-\frac{1}{5} h^{2} g_{, \alpha} Q_{\alpha}+A_{3333} h\left(\frac{17}{140} p^{2}+q^{2}\right)\right. \\
\left.+\frac{6}{5 h} A_{\alpha 3 \beta 3} Q_{\alpha} Q_{\beta}\right] d x_{1} d x_{2}, \\
\boldsymbol{d}^{\prime} \cdot \mathbf{\sigma}^{\prime \prime}=\int_{S}\left[\frac{2}{3} h^{3} B_{\alpha \beta \gamma \delta} w_{, \alpha \beta} w_{. \gamma \delta}+\frac{1}{5} h^{2}\left(g_{, \alpha} Q_{\alpha}+4 g p\right)\right] d x_{1} d x_{2} .
\end{array}
$$

Thus, the error in the stresses (5.1) is given by (1.12) and (5.2). Bounds on the relative error are given by (1.16) and (5.2).

By (3.8) and (3.11), (5.2) can be written as

$$
E^{2}=C_{1} h^{5}+C_{2} h^{7}, \quad \boldsymbol{\sigma}^{\prime} \cdot \mathbf{\sigma}^{\prime \prime}=C_{3} h^{3}+C_{4} h^{5},
$$

where $C_{1}, C_{2}, C_{3}, C_{4}$ depend only on $w$ and the elastic constants. Thus, by (5.3), we have

$$
\eta=E /\left(\mathbf{d}^{\prime} \cdot \mathbf{d}^{\prime \prime}\right)^{1 / 2}=C h+O\left(h^{3}\right),
$$

where

$$
\begin{aligned}
C=\left(\frac{C_{1}}{C_{3}}\right)^{1 / 2}= & \left\{\int _ { s } \left[\frac{1}{10} B_{\alpha \beta \beta 3} g_{, \alpha} g_{, \beta}-\frac{1}{15} g_{. \alpha} \widetilde{B}_{\alpha \beta \gamma \delta} w_{, \beta \gamma \delta}\right.\right. \\
& \left.\left.+\frac{4}{15} A_{\alpha \beta \beta 3} B_{\alpha \gamma \delta n} w_{, \gamma \delta n} B_{\beta \mu \xi \xi} w_{, \mu \zeta \xi}\right] d x_{1} d x_{2} / \int_{s} \frac{2}{3} B_{\alpha \beta \gamma \delta} w_{, \alpha \beta} w_{. \gamma \delta} d x_{1} d x_{2}\right\}^{1 / 2} .
\end{aligned}
$$


Equations (1.16) and (5.4) show that the relative error in the approximate stresses (5.1) is $O(h)$. This result is somewhat surprising in view of the elasticity solutions for plates [1] where corrections to classical plate theory are $O\left(h^{2}\right)$. A study of these solutions shows that the relative error of $O(h)$ in our formulas arises from the shear stress $\sigma_{\alpha \beta}^{\Lambda}$ in (5.1) whereas $\sigma_{\alpha \beta}^{A}$ and $\sigma_{33}^{A}$ contribute $O\left(h^{2}\right)$ to the error. Since $\sigma_{\alpha \beta}$ are usually the largest stresses in plate bending, (5.4) may be unduly pessimistic from a practical viewpoint.

Note added in proof: James G. Simmonds (in a paper accepted for publication in this journal) has improved the bound on the relative error to $O\left(h^{2}\right)$ for isotropic plates by use of a more elaborate displacement field than (3.1). This result appears to hold for anisotropic plates as well.

\section{REFERENCES}

[1] A. E. H. Love, A treatise on the mathematical theory of elasticity, 4th ed., Dover, New York, 1944

[2] J. N. Goodier, On the problems of the beam and the plate in the theory of elasticity, Trans. Roy. Soc. Canada (3) 32, 65-88 (1938)

[3] E. Reissner, On the derivation of boundary conditions for plate theory, Proc. Roy. Soc. Ser. A 276,178186 (1963)

[4] D. Morgenstern, Herleitung der Plattentheorie aus der dreidimensionalen Elastizitätstheorie, Arch. Rational Mech. Anal. 4, 145-152 (1959)

[5] I. Babuška and M. Prager, Reissnerian algorithms in the theory of elasticity, Bull. Acad. Polon. Sci. Sér. Sci. Math. Astr. Phys. 8, 411-417 (1960)

[6] M. Prager and J. L. Synge, Approximations in elasticity based on the concept of function space, Quart. Appl. Math. 5, 241-269 (1947)

[7] J. L. Synge, The hypercircle in mathematical physics: a method for the solution of boundary value problems, Cambridge Univ. Press, New York, 1957

[8] A. E. Taylor, Introduction to functional analysis, Wiley, New York, 1958 